Modeling of the refractive index and composition of luminescent nanometric chlorinated-silicon nitride films with embedded Si-quantum dots

A. Rodríguez-Gómez, L. Escobar-Alarcón, R. Serna, F. Cabello, E. Haro-Poniatowski, A. García-Valenzuela, and J. C. Alonso'

Citation: Journal of Applied Physics 120, 145305 (2016); doi: 10.1063/1.4964812

View online: http://dx.doi.org/10.1063/1.4964812

View Table of Contents: http://aip.scitation.org/toc/jap/120/14

Published by the American Institute of Physics

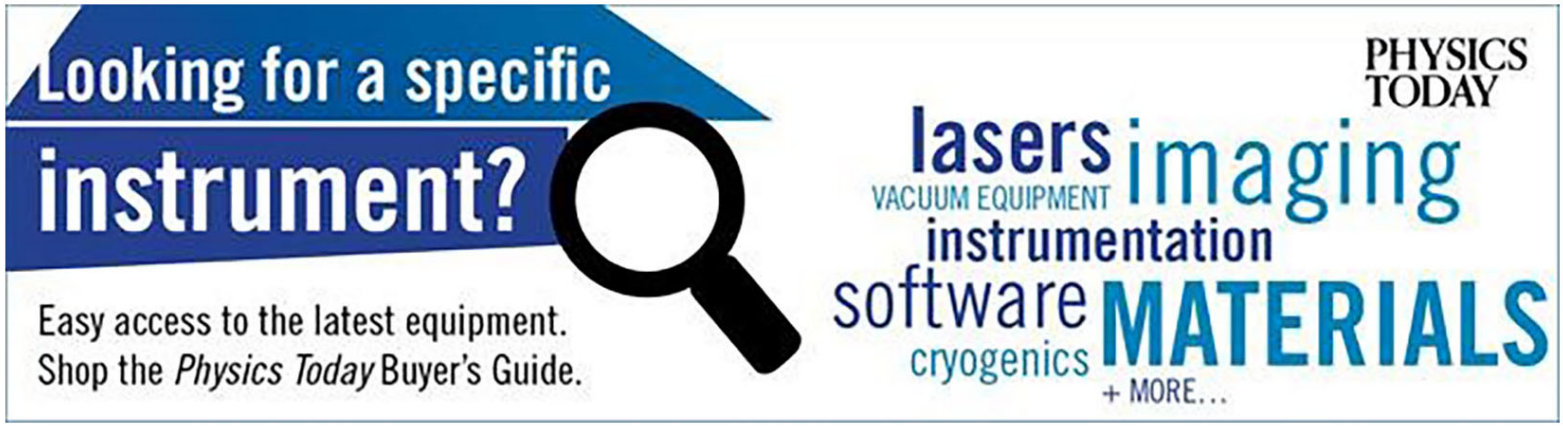




\title{
Modeling of the refractive index and composition of luminescent nanometric chlorinated-silicon nitride films with embedded Si-quantum dots
}

\author{
A. Rodríguez-Gómez, ${ }^{1}$ L. Escobar-Alarcón, ${ }^{2}$ R. Serna, ${ }^{3}$ F. Cabello, ${ }^{3}$ E. Haro-Poniatowski, ${ }^{4}$ \\ A. García-Valenzuela, ${ }^{5}$ and J. C. Alonso ${ }^{6, a)}$ \\ ${ }^{1}$ Instituto de Física, Universidad Nacional Autónoma de México, A:P. 20-364, Coyoacán 01000, \\ Ciudad de México, Mexico \\ ${ }^{2}$ Departamento de Física, Instituto Nacional de Investigaciones Nucleares, Apdo. Postal 181027, \\ Ciudad de México 11801, Mexico \\ ${ }^{3}$ Laser Processing Group, Instituto de Óptica, CSIC, Serrano 121, 28006 Madrid, Spain \\ ${ }^{4}$ Departamento de Física, Universidad Autónoma Metropolitana, Iztapalapa, \\ Av. San Rafael Atlixco No. 186, Col Vicentina, A.P. 09340, Ciudad de México, Mexico \\ ${ }^{5}$ Centro de Ciencias Aplicadas y Desarrollo Tecnológico, Universidad Nacional Autónoma de México, \\ Apartado Postal 70-186, Coyoacán 04510, Ciudad de México, Mexico \\ ${ }^{6}$ Instituto de Investigaciones en Materiales, Universidad Nacional Autónoma de México, \\ Apartado Postal 70-360, Coyoacán 04510, Ciudad de México, Mexico
}

(Received 5 August 2016; accepted 1 October 2016; published online 12 October 2016)

\begin{abstract}
The refractive index of nanometric $(<100 \mathrm{~nm})$ chlorinated-silicon nitride films with embedded silicon quantum dots, prepared by remote plasma enhanced chemical vapor deposition was investigated by spectroscopic ellipsometry. The complex refractive indexes and thicknesses of the films were obtained from the ellipsometric measurements using the Cauchy model. The chemical composition of the bulk of the films, including the hydrogen, chlorine, and oxygen content, was measured by elastic forward analysis (EFA) and X-ray photoelectron spectroscopy (XPS). The EFA and XPS results indicate that the films are composed of nearly stoichiometric silicon nitride $\left(\mathrm{Si}_{3} \mathrm{~N}_{4}\right)$ with different amounts of hydrogen, chlorine, and oxygen, and that their top surface is oxidized. Based on the composition results the refractive index of the films was fitted using the effective medium approximation considering the system: $\mathrm{Si}$ substrate $+\mathrm{Si}_{3} \mathrm{~N}_{4}+\mathrm{Si}+$ voids + top ultrathin oxidized surface layer. Published by AIP Publishing. [http://dx.doi.org/10.1063/1.4964812]
\end{abstract}

\section{INTRODUCTION}

Luminescent and down-converting silicon quantum dots (Si-QDs) embedded in silicon nitride and silicon dioxide thin films continue being of great interest to develop silicon based devices for photonic, optoelectronic, and photovoltaic applications. ${ }^{1-7}$ For several specific applications and investigations, the control and determination of the refractive index and composition of these films are very important. Spectroscopic ellipsometry has been widely used to measure the real and imaginary parts of the refractive index or dielectric function, and the thickness of different luminescent nanostructured silicon-rich silicon nitride films. ${ }^{5,8-13}$ Many of these cited works have shown that there is close correlation among the optical parameters of the films, and their composition, structure, and luminescent properties.

Based on the values of the real part of refractive index at $632 \mathrm{~nm}$ for pure $\mathrm{Si}(3.88), \mathrm{Si}_{3} \mathrm{~N}_{4}(2.0)$, and $\mathrm{SiO}_{2}$ (1.45), the relationships between molar composition and refractive index for silicon-silicon nitride and silicon-silicon oxide compounds can be predicted. ${ }^{5,14}$ Values progressively higher than 2.0 are expected for silicon rich silicon nitride $\left(\mathrm{SiN}_{\mathrm{x}}\right.$, $\mathrm{x}<1.33$ ) films as they become silicon richer and their atomic ratio $x=[\mathrm{N}] /[\mathrm{Si}]$ decreases. On the other hand, a linear decrease from 2.0 to 1.45 is expected in the values of the refractive index of silicon oxynitride as the oxygen content

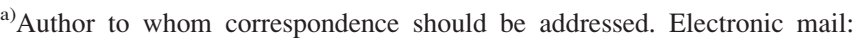
alonso@unam.mx
}

increases. ${ }^{14}$ However, the values and the empirical relationship between refractive index and $\mathrm{x}$ depend significantly on the composition of the films and the type and amount of other impurities, which in turn depend on the deposition method and experimental conditions. For example, for amorphous silicon rich silicon nitride films deposited by radio frequency magnetron sputtering which are free of hydrogen and oxygen, the refractive index (at $632.8 \mathrm{~nm}$ or $1.95 \mathrm{eV}$ ) increases from $\sim 1.83$ to 3 as $\mathrm{x}$, measured by Rutherford Back Scattering (RBS), decreases from 1.33 to $0.5 .^{5}$ For silicon rich silicon nitride films deposited by chemical vapor deposition techniques, which contain significant amounts of hydrogen, the refractive index increases but in a lower range from $\sim 1.8$ to 2.45 as $\mathrm{x}$, measured by $\mathrm{X}$-ray photoelectron spectroscopy (XPS), decreases from 1.0 to $0.5 .^{12}$ In a previous work, we have found that luminescent chlorinatedhydrogenated $\mathrm{SiN}_{\mathrm{x}}$ films with embedded silicon quantum dots, prepared by remote plasma enhanced chemical vapor (RPECVD) deposition, have $\mathrm{x}=[\mathrm{N}] /[\mathrm{Si}]$, measured by XPS, in the range from 1.16 to 0.7 , but, however, the refractive index of the films at $632 \mathrm{~nm}$ remains almost constant with low values $\sim 1.82-1.85$. $^{7,15,16}$ Based on the general trends mentioned above for the refractive index of silicon-silicon nitride and silicon-silicon oxide compounds, it seems contradictory that silicon rich silicon nitride films have refractive index values lower than 2.0. The explanation of the origin of the low refractive index of these films in terms of their composition was the main motivation for the present work. 
One question that complicates finding the correlation between the composition and refractive index of the films is that some of the most common techniques used to analyze the composition, such as XPS and RBS, do not detect hydrogen. ${ }^{17,18}$ Thus when the films contain hydrogen, other techniques, such as infrared spectroscopy (IR), elastic recoil detection analysis (ERDA) with light ions, or hydrogen forward scattering (HFS), have to be used to determine separately the hydrogen concentration. ${ }^{19,20}$ Although IR is a relatively inexpensive and common technique used to measure the $\mathrm{H}$ content, it requires a careful spectra interpretation in order to obtain reliable quantitative results; ${ }^{20}$ furthermore for quantitative analysis of the nanometric films, it is less reliable due to the very poor quality (low signal to noise ratio) of the IR spectra. ${ }^{15}$ Another complication with the analysis of the composition is that the SiNx films usually suffer oxidation when exposed to air and forms a silicon oxide layer or a graded oxynitride film. ${ }^{21}$ Since this oxidation reduces the amount of nitrogen at the film surface and since the XPS only identifies the chemical species at the top surface of the SiNx films, ${ }^{17}$ the $[\mathrm{N}] /[\mathrm{Si}]$ ratios determined by XPS will tend to be lower than the real $[\mathrm{N}] /[\mathrm{Si}]$ ratio in the bulk of the film. On the contrary, the RBS technique provides quantitative analysis of the elements in all the bulk of the films with thickness up to $1000 \mathrm{~nm}$, but the depth resolution is of the order of $10 \mathrm{~nm}$, which makes difficult to resolve a thin oxide layer. ${ }^{18}$ Additionally, in order to make a good correlation between refractive index and composition it is convenient to have information on the short range structure of silicon rich-SiN $\mathrm{x}_{\mathrm{x}}$ films, to decide which of the two extreme models is the adequate to describe this structure. ${ }^{22}$ According to the random mixture model (RMM), the $\operatorname{SiN}_{\mathrm{x}}$ film consists of separated phases of $\mathrm{Si}_{3} \mathrm{~N}_{4}$ and $\mathrm{Si}$ phases, whereas in the random bonding model (RBM) the $\mathrm{SiN}_{\mathrm{x}}$ film is considered as a statistical distribution of $\mathrm{Si}_{-} \mathrm{Si}_{4-\mathrm{n}} \mathrm{N}_{\mathrm{n}}(\mathrm{n}=0$, $1,2,3,4)$ bonds. $^{12,22}$

In this work, we have used the elastic forward analysis (EFA) technique, ${ }^{23}$ combined with XPS and spectroscopic ellipsometry to investigate the composition and refractive index of luminescent chlorinated-hydrogenated nanometric SiNx films $(\sim 80-90 \mathrm{~nm})$ with embedded silicon quantum dots, deposited by remote plasma enhanced chemical vapor (RPECVD) deposition. We used the XPS technique to investigate the depth of the oxide layer formed by oxidation of the top surface of the films, and EFA was used to measure the hydrogen content and bulk composition of the films. Based on the XPS and EFA results, the ellipsometric data were accurately fitted using the effective medium approximation (EMA), assuming that the bulk of the films is mainly composed of nearly stoichiometric silicon nitride, with $\mathrm{Si}$ and void phases embedded, and an ultrathin silicon oxide layer at the film top surface.

\section{EXPERIMENTAL}

For this study, a series of four silicon-rich silicon nitride films with approximately the same thickness but different size of Si-QDs were prepared using gas mixtures of $\mathrm{SiH}_{2} \mathrm{Cl}_{2} /$ $\mathrm{NH}_{3} / \mathrm{H}_{2} / \mathrm{Ar}$, and the RPECVD system reported previously. ${ }^{7}$
The films were deposited on the mirror polished face of single-crystalline (100) pieces of silicon wafers with resistivity of $200 \Omega \mathrm{cm}$. The substrate temperature for deposition was $300^{\circ} \mathrm{C}$. The flow rates of $\mathrm{SiH}_{2} \mathrm{Cl}_{2}, \mathrm{H}_{2}$, and $\mathrm{Ar}$ were kept constant at 5, 20, and $75 \mathrm{sccm}$, respectively. In order to change the size of the Si-QDs embedded in the films, and based on our previous experimental works, ${ }^{7,16}$ the flow rates of $\mathrm{NH}_{3}$ chosen for depositing each of the four different samples were 50 (S1_50), 100 (S2_100), 200 (S3_200), and 300 (S4_300) sccm. Also based on the knowledge of the deposition rate from our previous works, the table of colors of $\mathrm{Si}_{3} \mathrm{~N}_{4}$ films at various ranges of film thickness, ${ }^{24}$ and the direct naked eye view of the growing films, the deposition time was controlled for each film to obtain nanometric films with thickness in the range from 77 to $93 \mathrm{~nm}$ (blue color). After deposition, the thickness and refractive index of the films were measured at the fixed wavelength of 632.8 of the He-Ne laser of a Manual L117 ellipsometer, using an incidence angle of $70^{\circ}$. Spectroscopic ellipsometry measurements were further performed at room temperature at several incident angles $\left(45^{\circ}, 55^{\circ}, 65^{\circ}\right.$ and $\left.75^{\circ}\right)$ in the range from 300 to $1700 \mathrm{~nm}$ using a J. A. Woollam VASE spectroscopic ellipsometer. The simultaneous analysis of the spectroscopic ellipsometry and transmittance data was performed using the WVASE32 software (Woollam Co. Inc.). The X-ray Photoelectron Spectroscopy (XPS) measurements were made using a Jeol JS 9200 XPS. The spectra were acquired by exciting the films with an $\mathrm{Al}-\mathrm{K} \alpha \mathrm{X}$-ray source in the low and high-resolution regimes. Additionally, samples were characterized by elastic forward analysis (EFA); ${ }^{23}$ in this case, a 12.0 $\mathrm{MeV} \mathrm{C}^{2+}$ ion beam from a Tandem Van de Graff accelerator was used. The angle between the ion beam and the sample surface was fixed at $30^{\circ}$ while the angle between the detected particles and the incident ion beam was fixed at $45^{\circ}$.

\section{RESULTS AND DISCUSSION}

In order to determine the refractive index $(n)$ of the different films as a function of wavelength $(\lambda)$, the ellipsometric parameters $\Psi$ and $\Delta$ were measured for each sample at multiple incident angles in the reflection mode. The obtained data were analyzed using the WVASE32 software (Woollam Co. Inc.) that allows multiangle fitting of the spectra using the transfer matrix (Abelés) formalism and assuming a 3 layer model: Si substrate/film/surface layer. Fig. 1 shows the ellipsometric data and fit of $\Psi$ and $\Delta$ for sample S3_200. The fit was quite good and the MSE (mean square error) was very low: lower than 7 for all the samples. The experimental data were fitted to calculate the refractive index $n(\lambda)$ of the films, considering extinction coefficient $\kappa=0$ and using the model of Si substrate + the Cauchy dispersion relation for the film

$$
n(\lambda)=A+\frac{B}{\lambda^{2}}+\frac{G}{\lambda^{4}},
$$

where $A, B$, and $C$ are the fit coefficients.

Figure 2 shows the refractive index calculated in this way for the four samples. When $\kappa(\lambda) \neq 0$ was taken into account in the fittings, it had very low values; lower 


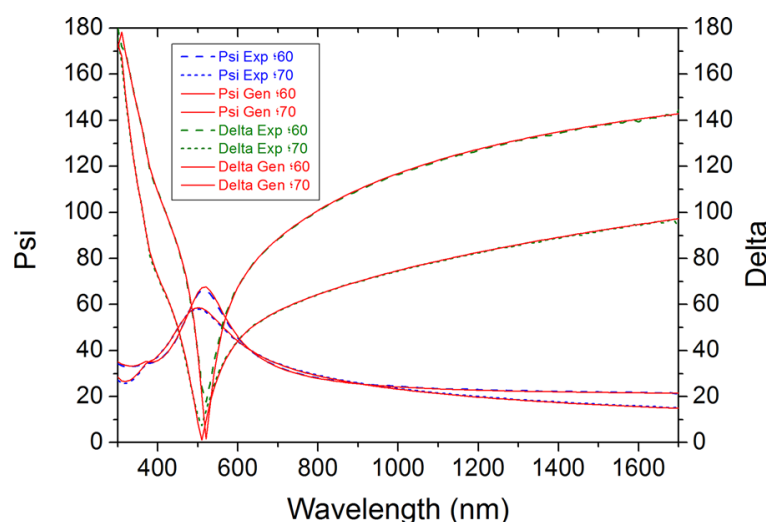

FIG. 1. Measured (dots) and fitted (lines) $\psi$ and $\Delta$ for sample S3_200.

than 0.001 , which are below the limit of resolution of ellipsometry.

Fig. 3 shows the XPS survey spectra of one of the samples (S3_200) without erosion and after $60 \mathrm{~s}$ of erosion with $2 \mathrm{keV}$ argon ions. In both spectra, it can be seen the presence of $\mathrm{Si}, \mathrm{N}, \mathrm{O}$, and $\mathrm{Cl}$. It is important to note that the signal corresponding to $\mathrm{O}$ is reduced significantly in the spectrum of the eroded film. It is also noticeable that the $\mathrm{C}$ signal disappears with the erosion, indicating that the presence of carbon is due to surface contamination. It is worth to mention that the XPS spectra of different samples were very similar.

Fig. 4 shows the depth profile of oxygen for the same sample S3, which reveals a fast decay of the oxygen content in the first $60 \mathrm{~s}$ of erosion, which indicates that the oxygen comes from oxidation of the film, and that the oxide surface layer attains a saturation thickness, which is very small compared with the film thickness.

Figure 5 shows the high resolution XPS spectra corresponding to the $\mathrm{O} 1 \mathrm{~s}$ peak without erosion and after $90 \mathrm{~s}$ of erosion. It is observed that the intensity of the $\mathrm{O} 1 \mathrm{~s}$ peak decreases and the position of the peak is shifted towards lower energies as a result of erosion time. The peak at $532.8 \mathrm{eV}$ observed in the spectrum of the sample without erosion, the surface of the film, is assigned to $\mathrm{O} 1 \mathrm{~s}$ in $\mathrm{SiO}_{2}$. This result indicates that the oxygen in/and close to the surface of the film is bonded to $\mathrm{Si}$ atoms forming $\mathrm{SiO}_{2}$. After

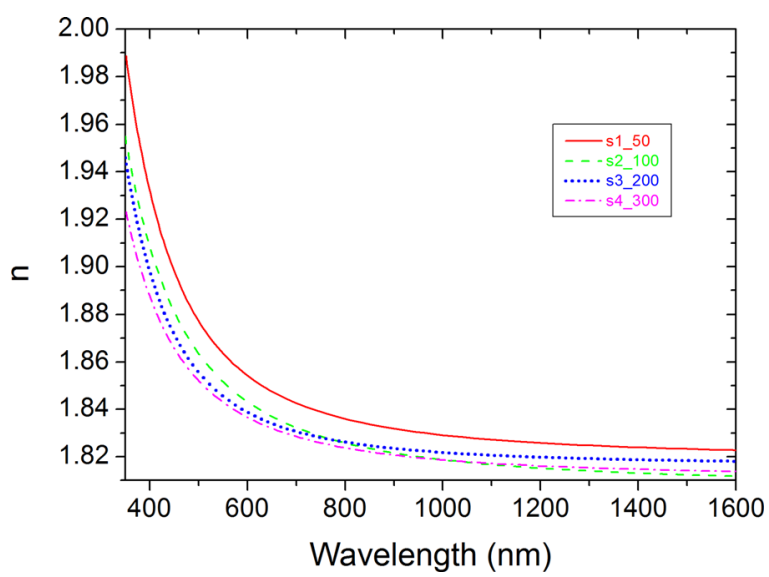

FIG. 2. Refractive index spectra for all the samples, calculated using the Cauchy dispersion relation.

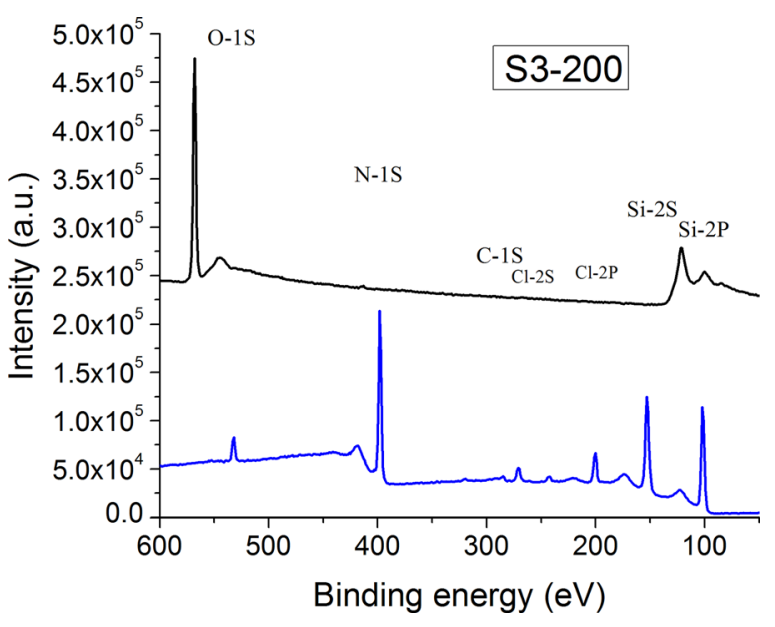

FIG. 3. XPS survey spectra of sample S3_200 without erosion (top) and after $60 \mathrm{~s}$ of erosion (bottom) with $2 \mathrm{keV}$ argon ions.

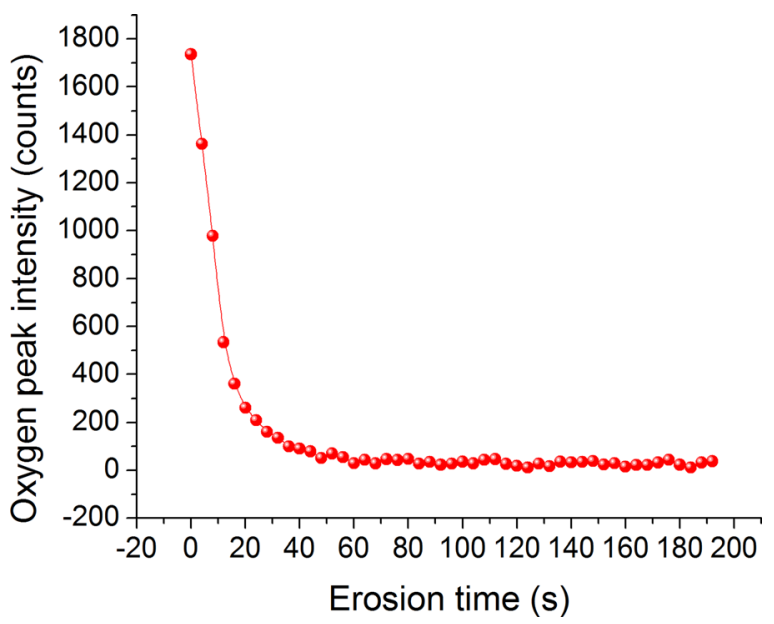

FIG. 4. XPS depth profile of oxygen for the same sample S3_200.

erosion, the peak appears at $532.1 \mathrm{eV}$ attributed to bonds $\mathrm{O}-\mathrm{Si}$ as well as to $\mathrm{O} 1 \mathrm{~s}$ in $\mathrm{Si}(\mathrm{OH})_{4}$ suggesting that in the interior of the film it seems to be incorporated as $\mathrm{OH}$ in a very small amount.

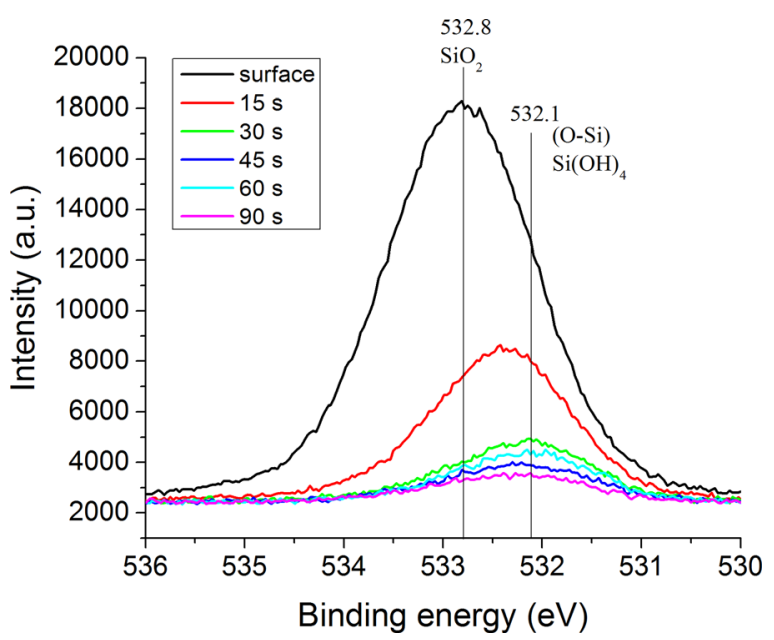

FIG. 5. High resolution XPS spectra corresponding to the O 1s peak for the sample S3_200 without erosion and after different times of erosion. 


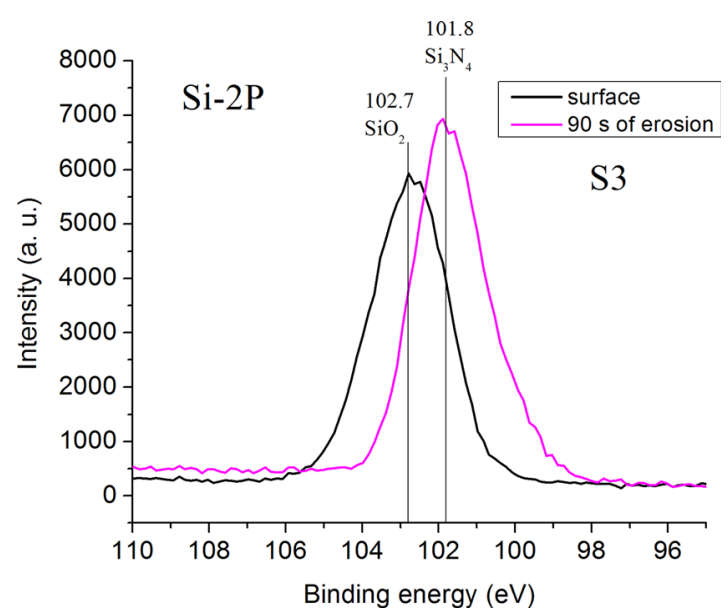

FIG. 6. High resolution XPS spectra corresponding to the Si 2p peak for sample S3_200 without erosion and after 90 s of erosion.

The high resolution XPS spectra corresponding to Si $2 p$ is shown in Fig. 6. From this figure, one can see that at the surface and until a depth corresponding to $90 \mathrm{~s}$ the $\mathrm{Si}$ atoms are mainly oxidized indicating again that there is a surface layer of $\mathrm{SiO}_{2}$, and in the interior of the film, there is $\mathrm{Si}_{3} \mathrm{~N}_{4}$ in higher proportion.

Figure 7 shows the resolved N 1s XPS spectra, in which it is observed that the nitrogen atoms are bonded to $\mathrm{Si}$ atoms forming $\mathrm{Si}_{3} \mathrm{~N}_{4}$. However, the amount of $\mathrm{N}$-bonded to $\mathrm{Si}$ is lower at the surface and increases with the depth inside the film indicating again that some $\mathrm{N}-\mathrm{Si}$ bonds are replaced by $\mathrm{O}-\mathrm{Si}$ bonds due to the oxidation of the film surface. Additionally, it is observed a signal around $403.4 \mathrm{eV}$ attributed to $\mathrm{NH}_{4}$, indicating the presence of $\mathrm{H}$ into the film.

Fig. 8 shows the EFA spectra for samples S3_200 and S4_300. Besides the signals of the elements $\mathrm{Si}, \mathrm{N}, \mathrm{O}, \mathrm{Cl}$, the signal due to $\mathrm{H}$ is clearly observed. The presence of carbon could be attributed to backscattered $\mathrm{C}$ ions used as projectile.

The composition of all the films calculated from the EFA measurements is given in Table I. The uncertainty of these measurements was $\pm 15 \%$ for our experimental conditions.

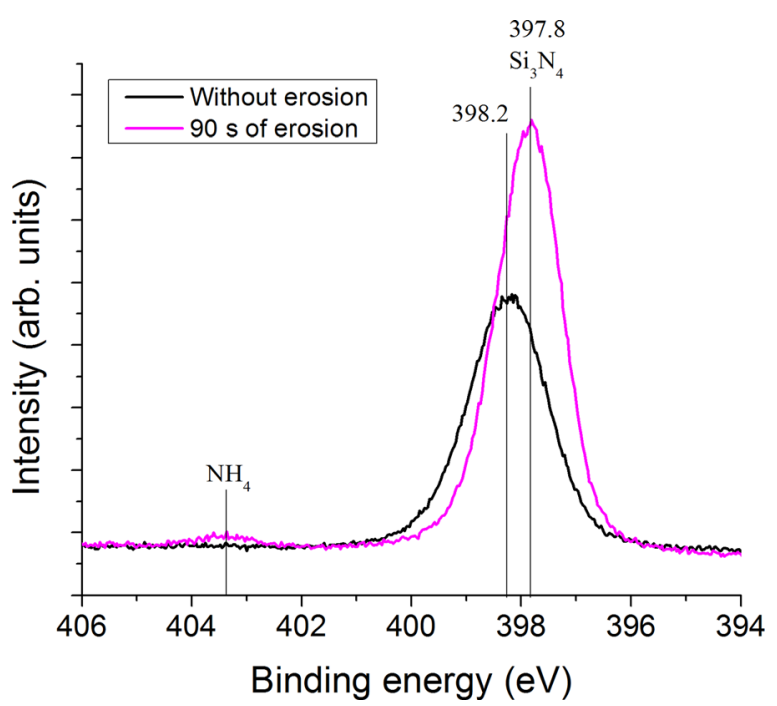

FIG. 7. High resolution spectra of the N 1s peak for sample S3_200 without erosion and after $90 \mathrm{~s}$ of erosion.

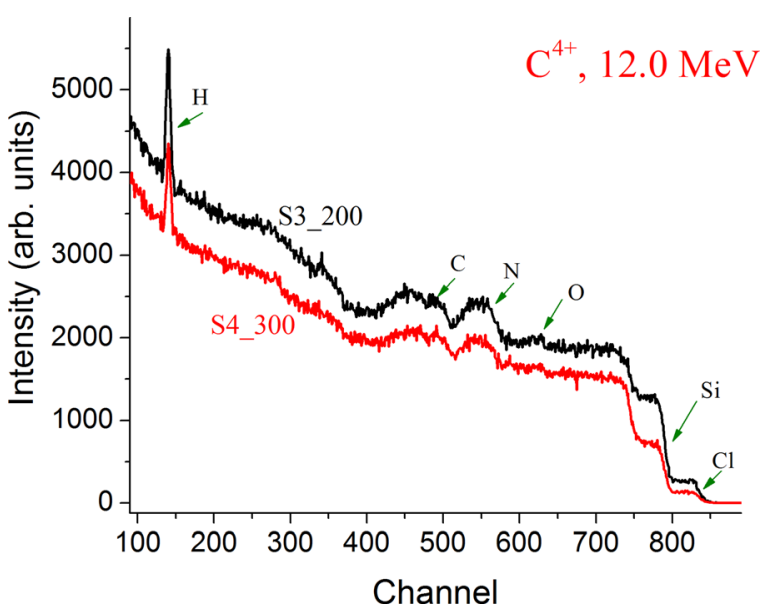

FIG. 8. EFA spectra for samples S3_200 and S4_300 using projectiles of $\mathrm{C} 4+$ of $12.0 \mathrm{MeV}$.

According to Table I, although it is apparent that the N/Si ratio increases slightly from sample $\mathrm{S} 1$ to sample $\mathrm{S} 4$, the average of the N/Si ratio for all the films is $N / S i \approx 1.35 \pm 0.15$, which, given the uncertainty of the measurements, correspond to that of stoichiometric silicon nitride $(N / S i \approx 1.33)$. Thus, the XPS and EFA results indicate that the bulk of all the films in average can be considered as composed of nearly stoichiometric silicon nitride $\left(\mathrm{Si}_{3} \mathrm{~N}_{4}\right)$, containing a significant amount of hydrogen ( $\sim 12-17$ at. \%), and some chlorine ( 4 at. \%) and oxygen as $\mathrm{OH}(\sim 2$ at. \%). Since all these elements or species form terminal bonds ( $\mathrm{Si}-\mathrm{H}, \mathrm{Si}-\mathrm{Cl}, \mathrm{Si}-\mathrm{OH}$ ), we can assume that they generate nanovoids or nanopores inside the $\mathrm{Si}_{3} \mathrm{~N}_{4}$ matrix, in a similar way as the $\mathrm{Si}-\mathrm{F}$ bonds generate nanopores in fluorinated $\mathrm{SiO}_{2}$ films. ${ }^{25,26}$ On the other hand, the HRTEM images of all these films indicate the presence of silicon nanoparticles. ${ }^{7}$ Based on all the preceding results, we assumed that the random mixture model (RMM) is the adequate to describe the short range structure of our $\mathrm{SiN}_{\mathrm{x}}$ films. As a consequence of this and to model their refractive index, they can be considered to consist of separated phases of $\mathrm{Si}_{3} \mathrm{~N}_{4}, \mathrm{Si}$ phases, and voids, as shown in Fig. 9.

Based on the XPS results that indicate that all the silicon nitride films suffer surface oxidation, the refractive index of the films were fitted using the Maxwell-Garnet expression of the effective medium approximation (EMA), ${ }^{27}$ considering the system: $\mathrm{Si}_{3} \mathrm{~N}_{4}+\mathrm{Si}+$ voids + (top ultrathin oxidized surface layer). In this approach, we considered $\mathrm{Si}_{3} \mathrm{~N}_{4}$ as the host material and $\mathrm{Si}$ and voids as the diluted phases. The refractive indices used for $\mathrm{Si}_{3} \mathrm{~N}_{4}$ and $\mathrm{Si}$ were 2.0 and 3.85, respectively. Although by ellipsometry it is not possible to distinguish if the nanovoids are filled with hydrogen, oxygen, or air, we used the \% of void in the EMA model to indicate

TABLE I. Composition of the films calculated from the EFA analysis.

\begin{tabular}{lcccccc}
\hline \hline Sample & Si (at. \%) & N (at. \%) & Cl (at. \%) & O (at. \%) & H & N/Si \\
\hline S1_50 & 35 & 41 & 4 & 3 & 17 & 1.2 \\
S2_100 & 34 & 43 & 4 & 2 & 17 & 1.3 \\
S3_200 & 33.5 & 47 & 3.5 & 1 & 15 & 1.4 \\
S4_300 & 33.5 & 48 & 4.5 & 1 & 12 & 1.5 \\
\hline \hline
\end{tabular}




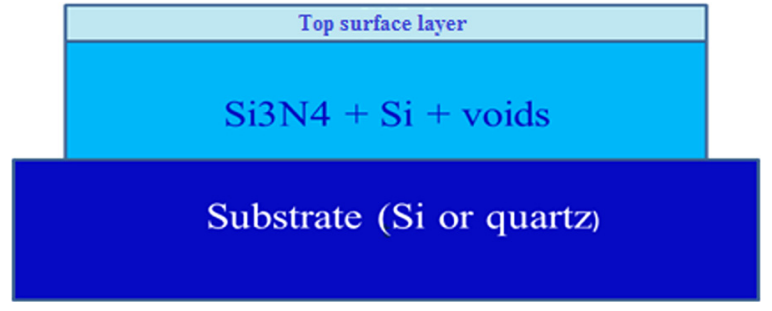

FIG. 9. Model used to correlate the refractive index of the films with their composition using the effective medium approximation.

TABLE II. Thickness of the surface and EMA layers, and percentages of $\mathrm{Si} 3 \mathrm{~N} 4, \mathrm{Si}$, and voids obtained from the EMA model. MSE is the mean square error of the fits.

\begin{tabular}{lcccc}
\hline \hline & S1_50 & S2_100 & S3_200 & S4_300 \\
\hline Thickness (nm) surface layer & 5.9 & 4.9 & 5.1 & 3.3 \\
Thickness (nm) EMA layer & 77.7 & 91.2 & 76.7 & 84.9 \\
\%Si3N4 & 74.5 & 77.0 & 79.9 & 80.8 \\
\%VOID & 21.8 & 20.8 & 19.1 & 18.9 \\
\%Si & 3.8 & 2.2 & 1.0 & 0.3 \\
MSE & 7 & 2 & 3 & 5 \\
\hline \hline
\end{tabular}

that we have a percentage of diluted material with a refractive index equal to 1.0. Under the previous assumption on the composition of the films and refractive indices of the host and diluted phases, the fit was quite good for all the samples. Table II shows the percentage of each EMA region for each film obtained from the fits. The high percentages of $\mathrm{Si}_{3} \mathrm{~N}_{4}$ in this table indicates that this is the host medium of the films, in which the void and $\mathrm{Si}$ phases are embedded in smaller proportion. As expected, the percentage of $\mathrm{Si}_{3} \mathrm{~N}_{4}$ increases with the decrease in the percentage of $\mathrm{Si}$, as the flow rate of $\mathrm{NH}_{3}$ used during deposition is increased. The at. $\%$ of Si and $\mathrm{N}$ was estimated using the data of Table II and the formulas: at. $\% \mathrm{Si}=3 / 7\left(\% \mathrm{Si}_{3} \mathrm{~N}_{4}\right)+\% \mathrm{Si}$ and at. $\% \mathrm{~N}=4 / 7$ $\left(\% \mathrm{Si}_{3} \mathrm{~N}_{4}\right)$. The obtained results are shown in Table III.

The data of Table II also show that the $\%$ of the void content in the films decreases from sample S1_50 to sample S4_300. It should be noted that, comparing the data of Tables I and II, the void percentage is in good agreement with the percentage of $(\mathrm{H}+\mathrm{O}+\mathrm{Cl})$ measured by EFA. On the other hand, according to the data of Table III, the relative silicon excess decreases and the nitrogen content increases from sample S1_50 to sample S4_300, and this behavior is expected since the flow rate of the $\mathrm{NH}_{3}$ increases from sample S1_50 to sample S4_300. The apparent decrease in the thickness of the ulthrathin oxide surface layer observed in Table II is consistent with the decrease in the oxygen content

TABLE III. At. $\%$ of $\mathrm{Si}$ and $\mathrm{N}$ for the films, considering the $\mathrm{Si}_{3} \mathrm{~N}_{4}$ matrix and the Si phase embedded as silicon quantum dots in this matrix.

\begin{tabular}{lcccc}
\hline \hline & S1_50 & S2_100 & S3_200 & S4_300 \\
\hline $\mathrm{Si}$ & 35.7 & 35.2 & 35.2 & 34.9 \\
$\mathrm{~N}$ & 42.6 & 44.0 & 45.7 & 46.2 \\
$\mathrm{~N} / \mathrm{Si}$ & 1.19 & 1.25 & 1.30 & 1.32 \\
\hline \hline
\end{tabular}

in the bulk of the films observed in Table I, as going from sample S1_50 to sample S4_300, and indicates that the films are more resistant to post-deposition oxidation as the $\mathrm{NH}_{3}$ increases.

\section{CONCLUSION}

We can conclude that the low refractive index of the films, despite that they are silicon rich, is due the large percentage of voids in the bulk of the films. The invariability of the refractive index among these films, despite that they were deposited at different $\mathrm{NH}_{3}$ flow rates, is due to the fact that as they become silicon rich they contain a higher amount of voids, so the increase in the refractive index of the films due to the increase of the $\mathrm{Si}$ phase content is compensated with the decrease in the refractive index due to the increase of the content of voids. Finally, it is observed that the ultrathin oxide layer formed at the surface of the films by post deposition oxidation has negligible influence on their optical properties.

\section{ACKNOWLEDGMENTS}

The authors want to acknowledge the technical assistance of J. M. García-León. This research work was partially supported under Project PAPIIT-UNAM No. IG100614-2. We also acknowledge the funding from the Spanish Ministry for Economy and Competitiveness through TEC 2012-38901C02-01 and TEC 2015-69916-C2-1-R projects.

${ }^{1}$ B. Sain and D. Das, J. Lumin. 158, 11 (2015).

${ }^{2}$ F. Priolo, T. Gregorkiewicz, M. Galli, and T. F. Krauss, Nat. Nanotechnol. 9, 19 (2014).

${ }^{3}$ O. Blázquez, J. López-Vidrier, S. Hernández, J. Montserrat, and B. Garrido, Energy Procedia 44, 145 (2014).

${ }^{4}$ D. Hiller, A. Zelenina, S. Gutsch, S. A. Dyakov, L. López-Conesa, J. López-Vidrier, S. Estradé, F. Peiró, B. Garrido, J. Valenta, M. Kořínek, F. Trojánek, P. Malý, M. Schnabel, C. Weiss, S. Janz, and M. Zacharias, J. Appl. Phys. 115, 204301 (2014).

${ }^{5}$ O. Debieu, R. P. Nalini, J. Cardin, X. Portier, J. Perrière, and F. Gourbilleau, Nanoscale Res. Lett. 8, 31 (2013).

${ }^{6}$ W. R. Taube, A. Kumar, R. Saravanan, P. B. Agarwal, P. Kothari, B. C. Joshi, and D. Kumar, Sol. Energy Mater. Sol. Cells 101, 32 (2012).

${ }^{7}$ A. Rodriguez, J. Arenas, and J. C. Alonso, J. Lumin. 132, 2385 (2012).

${ }^{8}$ A.-S. Keita, A. E. Naciri, F. Delachat, M. Carrada, G. Ferblantier, and A. Slaoui, J. Appl. Phys. 107, 093516 (2010).

${ }^{9}$ P. Basa, P. Petrik, M. Fried, L. Dobos, B. Pécz, and L. Tóth, Phys. E 38, 76 (2007).

${ }^{10}$ Z. H. Cen, T. P. Chen, L. Ding, Y. Liu, M. Yang, J. I. Wong, Z. Liu, Y. C. Liu, and S. Fung, Appl. Phys. Lett. 93, 023122 (2008).

${ }^{11}$ V. E. Vamvakas, N. Vourdas, and S. Gardelis, Microelectron. Reliab. 47, 794 (2007).

${ }^{12}$ J. F. Lelièvre, E. Fourmond, A. Kaminski, O. Palais, D. Ballutaud, and M. Lemiti, Sol. Energy Mater. Sol. Cells 93, 1281 (2009).

${ }^{13}$ V. E. Vamvakas and S. Gardelis, Surf. Coatings Technol. 201, 9359 (2007).

${ }^{14}$ T. Bååk, Appl. Opt. 21, 1069 (1982).

${ }^{15}$ A. Rodriguez-Gómez, A. García-Valenzuela, E. Haro-Poniatowski, and J. C. Alonso-Huitrón, J. Appl. Phys. 113, 233102 (2013).

${ }^{16}$ A. Rodríguez, J. Arenas, A. L. Pérez-Martínez, and J. C. Alonso, Mater. Lett. 125, 44 (2014)

${ }^{17}$ B. D. Ranter and D. G. Castner, in Surface Analysis: The Principal Techniques, edited by John C. Vickerman (Wiley, 2002), pp. 43-98.

${ }^{18}$ E. Taglauer, in Surface Analysis: The Principal Techniques, edited by J. C. Vickerman (Wiley, 2002), pp. 215-266. 
${ }^{19}$ W. Bohne, W. Fuhs, J. Rohrich, B. Selle, G. Gonzalez-Diaz, I. Martil, F. L. Martinez, and A. del Prado, Surf. Interface Anal. 30, 534 (2000).

${ }^{20}$ G. Morello, J. Non-Cryst. Solids 187, 308 (1995).

${ }^{21}$ S. I. Raider, R. Flitsch, J. A. Aboaf, and W. A. Pliskin, J. Electrochem. Soc. 123, 560 (1976).

${ }^{22}$ V. A. Gritsenko, R. W. M. Kwok, H. Wong, and J. B. Xu, J. Non-Cryst. Solids 297, 96 (2002)
${ }^{23}$ S. Romero, M. Fern, G. Murillo, and H. M. Berdejo, Nucl. Instrum. Methods Phys. Res. 194, 164 (2002).

${ }^{24}$ W. V. G. F. Reizman, Solid State Electron. 10, 625 (1967).

${ }^{25}$ J. C. Alonso, X. M. Díaz-Bucio, E. Pichardo, L. Rodríguez-Fernández, and A. Ortiz, Thin Solid Films 474, 294 (2005).

${ }^{26}$ J. H. Lee and C. K. U. Hwangbo, Surf. Coatings Technol. 128-129, 280 (2000).

${ }^{27}$ D. E. Aspnes, Thin Solid Films 89, 249 (1982). 